\title{
A Cross-Hierarchical Scanning Method Based SP-4-WFRFT for Digital Communication Signals
}

\author{
Fang Liu \\ School of Information Science and Engineering, Shenyang Ligong University, Shenyang 110159, China \\ Correspondence should be addressed to Fang Liu; zhqing1019@163.com
}

Received 6 June 2018; Accepted 30 July 2018; Published 8 August 2018

Academic Editor: Luigi Rodino

Copyright ( 2018 Fang Liu. This is an open access article distributed under the Creative Commons Attribution License, which permits unrestricted use, distribution, and reproduction in any medium, provided the original work is properly cited.

\begin{abstract}
The fractional Fourier transform (FRFT) method has emerged in response to the demand for high-performance time-frequency processing technology. Next, the weighted fractional Fourier transform (WFRFT) method was developed, especially the single-parameter 4-weighted fractional Fourier transform (SP-4-WFRFT), which is most suitable for applications in digital communication systems. Therefore, in receiving systems, research on the scanning method of transformation order has also become the focus of research under the condition of unknown transformation order. However, in the process of inverse transformation, there are many problems, such as long scanning times and slow scanning speed. Thus, to improve the characteristics of a digital communication system based on SP-4-WFRFT, a cross-hierarchical scanning (CHS) method is established. A large number of test results show that the CHS method can achieve fast recovery of data and estimation of transformation order.
\end{abstract}

\section{Introduction}

Fourier transform can be regarded as the relationship between time domain and frequency domain, and the timefrequency plane is rotated $\pi / 2$. Fourier transform provides a method of time domain and frequency domain conversion, but it can only be carried out in one of the domains in signal processing, and the change of the spectrum with time cannot be obtained, which is very important for nonstationary signals because the spectrum varies greatly with time. Thus, a series of time-frequency analysis methods have been proposed, such as short time Fourier transform, wavelet transform, and the later fractional Fourier transform (FRFT).

The concept of FRFT was proposed by Wiener in 1929 [1]. The definition of FRFT is that the rotation angle is not integer multiple of pi/2 in the time-frequency plane. Its purpose is to modify the ordinary Fourier transform to make the transform kernel's eigenvalues more complete, so FRFT is a generalization of the traditional Fourier transform. Its basic characteristics can be understood as the fractional transformation of the eigenvalues of Fourier transform. That is to say, the signal rotates anticlockwise at any angle in the time domain plane and maps onto the fractional
Fourier domain. Because of the different way of eigenvalue fractionation and the broad nature of FRFT constraints, FRFT has many different definitions. CFRFT (Chirp-type fractional Fourier transform) is an earlier form of FRFT, and there are mainly three forms. The first form is the fractional Fourier transformation from the point of view of the eigenvalues and characteristic functions of Fourier transformation introduced in 1980, which is represented mathematically as the form of "infinite series sum" [2]. The next is the "integral form" [3] proposed by A. C. McBride and F. H. Kerr based on Namias's standard chirp-type fractional Fourier transform introduced in 1987. The third form is the fractional Fourier transform defined by A. W. Lohmann in 1993 from the angle of the phase space of Wigner distribution function [4]. The three definitions are different in terms of research, but they can be proved to be equivalent.

Classical FRFT has been widely applied in various scientific research areas, such as differential equations, quantum mechanics, and signal analysis and processing. Engineering technologies, such as optical communication systems, optical image processing, and other optical related fields, are the earliest scientific fields to use classic FRFT, and they are also the most successful applications at present. 
However, constrained by the discrete problem of classical FRFT, its application in the field of communication was greatly restricted. Then C. C. Shih proposed the weighted fractional Fourier transform (WFRFT) [5] from a new angle in 1995. This transformation is composed of the weighted superposition of the state functions, and it is also called the four-term weighted fractional Fourier transform because the number of state functions is 4. In 1997, M. H. Ozaktas and M. A. Kutar proposed optimal filtering in the fractional domain, which allowed the application of FRFT in signal processing for the first time [6]. Then, J. Jiang, S. T. Liu, and others studied FRFT theory more deeply, perfected the definition of WFRFT, and proposed a multiplier of FRFT with a weighted transformation period of 4 ; they gave the detailed construction method of the weighted coefficient under this definition [7]. In 2008, G. Cariolaro and Q. W. Ran unified the above several forms of fractional Fourier transform and then defined a generalized multiparameter Fourier transform $[8,9]$.

In recent years, WFRFT has been widely used in the fields of signal detection, channel estimation, and synchronization algorithms, and it is fully applicable to the existing communication and receiving systems. It does not require additional devices or system changes, so it is also applied in many communication applications. In [10], the FRFT was first introduced for satellite navigation signal processing to obtain the time offset and Doppler shift directly through the different angles of the fractional Fourier transform. In [11], The FRFT is applied to the detection of high dynamic targets. In [12], satellite signal is processed by MAP-WFRFT to rotate and diverge the distribution of the signal constellation, disguised as noise or another modulation mode, thus realizing the concealment of the signal. In [13], WFRFT is used to preprocess transmitted signals, and a new physicallayer security communication method is proposed. In [14], based on the analysis of the sample and hold system in the fractional Fourier domain, a feasible engineering sampling and reconstructing model is proposed. In [15], a new method of central frequency estimation is proposed based on the FRFT, which is based on the time width of the wideband LFM pulse and the observation time width.

Obviously, WFRFT has become an important application of digital communication systems, and the SP-4-WFRFT (single-parameter 4-weighted fractional Fourier transform) is the most widely used. However, because of the high sensitivity of the transformation order, to restore the original data correctly, the transformation order of the inverter is more demanding, especially when there is error or influence in the application of the communication system. Therefore, in the process of fractional Fourier inverse transformation, the scanning method of transformation order has also become the focus of research. At present, most people use SP-4WFRFT method to transmit signals under the condition of known parameter, but there are few scanning methods for unknown parameters. And the conventional scanning (CS) method $[16,17]$ is the most effective method at present. Therefore, considering the characteristics of a digital communication system based on SP-4-WFRFT, an effective scanning method is proposed.

\section{The Problems in Scanning Progressing}

In digital communication systems, the transmission baseband signal is set to $s(n)$. The base-band signal is processed by an $\alpha$-order SP-4-WFRFT and is expressed as

$$
\begin{aligned}
F^{\alpha}(s(n))= & \omega_{0}(\alpha) s(n)+\omega_{1}(\alpha) S(n)+\omega_{2}(\alpha) s(-n) \\
& +\omega_{3}(\alpha) S(-n) .
\end{aligned}
$$

The $\alpha$-order SP-4-WFRFT [16] processing is denoted by $F^{\alpha}(\cdot)$. In (1), the four "state functions" of $s(n), S(n), s(-n)$, and $S(-n)$ are the results of $0,1,2$, and 3 times Fourier transformation of $s(n)$ respectively. The weighting coefficient $\omega_{l}(\alpha)$ is defined as

$$
\begin{aligned}
\omega_{l}(\alpha)= & \cos \left[\frac{(\alpha-l) \pi}{4}\right] \cos \left[\frac{(\alpha-l) 2 \pi}{4}\right] \\
& \cdot \exp \left[\frac{j(\alpha-l) 3 \pi}{4}\right] .
\end{aligned}
$$

In combination with the formula DFT (Discrete Fourier transform), formula (1) can be equivalent to

$$
\begin{aligned}
F^{\alpha}(s(n))= & \omega_{0}(\alpha) s(n) \\
& +\omega_{1}(\alpha) \frac{1}{\sqrt{N}} \sum_{n=0}^{N-1} s(k) e^{-j(2 \pi / N) k n} \\
& +\omega_{2}(\alpha) s(-n) \\
& +\omega_{3}(\alpha) \frac{1}{\sqrt{N}} \sum_{n=0}^{N-1} s(k) e^{j(2 \pi / N) k n}
\end{aligned}
$$

In receiving, the base-band signal $r(n)$ after the frequency downconversion, power amplifying, and ADC and band-pass filtering can be set to formula (4). Here, $\lambda_{0}(n)$ represents the mixed noise item caused by the transmission process and receiving pretreatment:

$$
r(n)=F^{\alpha}(s(n))+\lambda_{0}(n) .
$$

Because Fourier transform has a cycle of 4 , with the change of $\alpha$, the weighting coefficient $\omega_{l}(\alpha)$ also presents a cycle of 4 . Further, $F^{\alpha}(\cdot)$ also meets the cycle characteristics, where the period of $\alpha$ is [ 04 ).

Since $r(n)$ is the signal after SP-4-WFRFT processing, to restore the data signal or estimate the transformation order $\alpha$, the inverse transformation of the received signal is carried out by using the rotational additive property of the SP-4-WFRFT transform. The inverse transformation is also equivalent to the SP-4-WFRFT transformation of the $-\beta$ order, which is expressed as

$$
\begin{aligned}
r^{\prime}(n) & =F^{-\beta}(r(n))=F^{-\beta}\left(F^{\alpha}(s(n))+\lambda_{0}(n)\right) \\
& =F^{-\beta}\left(F^{\alpha}(s(n))\right)+F^{-\beta}\left(\lambda_{0}(n)\right) \\
& =F^{\alpha-\beta}(s(n))+\lambda_{0}^{\prime}(n)
\end{aligned}
$$


In (5), when $\beta$ is equal to $\alpha$, the true signal of transmission can be correctly received or restored. However, to meet the requirements of the $\beta=\alpha$ equation under the condition that the transformation order is unknown, the aim of $\alpha$ scanning is achieved by the way of $\beta$ scanning in the main period

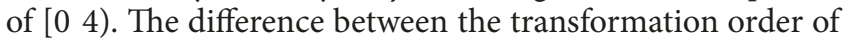
inverse transformation and positive transformation is $\Delta \beta=$ $\alpha-\beta$. Combined with the relationship between the scan interval and the bit error rate, the smaller $\Delta \beta$, the smaller the bit error rate of the signal after the inverse transform, that is, the smaller the error between $r^{\prime}(n)$ and real signal. However, the smaller $\Delta \beta$ is, the more time it takes to scan in the $0-4$ interval. For example, when $\Delta \beta=0.01$, the number of scanning cycles is 400 ; when $\Delta \beta=0.0078125$, the number of scanning cycles is 512 .

\section{A Cross-Hierarchical Scanning Method}

In the process of inverse transformation, under the condition of unknown transformation order $\alpha$, there are many problems, such as long scanning times and slow scanning speed. Thus, aiming at the characteristics of a digital communication system based on SP-4-WFRFT, a cross-hierarchical scanning (CHS) method is proposed to quickly recover data signals or estimate transformation order.

The maximum hierarchical constant is defined as $M$, which is the maximum hierarchical limit. Here, $M$ is a positive integer defined by the user according to the accuracy requirement, whose value is greater than or equal to 1 . Then the hierarchical variable is defined as $m, m \in[1,2 \cdots M]$, and $m$ increases from 1 to $M$. The scan variable is defined as $i$.

By using the hierarchical variable $m$, the scan number at each level is shown in formula (6), which is written as $L_{m}$. For example, when $m$ is equal to 1 , it indicates that it is the firstlevel scan, and its priority is the highest; the scan number is calculated as $L_{m}=2^{2}=4$ :

$$
L_{m}= \begin{cases}2^{2}, & (1 \leq m \leq 2) \\ 2^{m}, & (3 \leq m \leq M) .\end{cases}
$$

According to the scan number result, the ergodic range of the scan variable $i$ is limited. Furthermore, to calculate the transformation order $\beta$, the hierarchical rule is set as follows.

When $m \leq 2$, the ergodic range of $i$ is $i \in\left[\begin{array}{ll}1 & L_{m}\end{array}\right]$, and the hierarchical rule is expressed by (7). When $3 \leq m \leq M$, the ergodic range of $i$ is $i \in\left[\begin{array}{ll}1 & L_{m-1}\end{array}\right]$, and the hierarchical rule is expressed by (8). By using the hierarchical rules of formulae (7) and (8), the transformation order $\beta$ is obtained. Then, $\beta$ is used to scan the level $m$, which means that the received signal $r(n)$ is processed by SP-4-WFRFT inverse transform $L_{m}$ times, and the results of $L_{m}$ group processing are obtained.

$$
\begin{aligned}
\beta_{i}^{m} & = \begin{cases}j-1, & (m=1) \\
\beta_{i}^{m-1}+2^{1-m}, & (m=2)\end{cases} \\
\beta_{2 i-1}^{m} & =\beta_{i}^{m-1}-2^{1-m} \\
\beta_{2 i}^{m} & =\beta_{i}^{m-1}+2^{1-m} .
\end{aligned}
$$

Then the SP-4-WFRFT inverse transform of the $\beta$ order is expressed as

$$
\begin{aligned}
F^{-\beta}(r(n))= & \omega_{0}(-\beta) r(n)+\omega_{1}(-\beta) R(n) \\
& +\omega_{2}(-\beta) r(-n)+\omega_{3}(-\beta) R(-n) .
\end{aligned}
$$

The unified weighting coefficient $\omega_{l}(\beta)$ can be written as

$$
\omega_{l}(-\beta)=\frac{1}{4} \sum_{k=0}^{3} \exp \left[\frac{2 \pi j}{4}(l-\beta) k\right] \quad(l=0,1,2,3)
$$

For example, when $m$ is equal to 2 , it indicates that it is the second-level scan, and its priority is the second highest. The scan number is calculated as $L_{m}=2^{2}=4$, and the ergodic range of $i$ is $i \in\left[\begin{array}{ll}1 & 4\end{array}\right]$. Then by using the hierarchical rules of (7), the transformation order $\beta_{i}^{2}=\beta_{i}^{m-1}+2^{1-m}=$ $i-1+2^{-1}=i-2^{-1}$ is obtained. Using $\beta_{i}^{2}$, the received signal $r(n)$ is processed by SP-4-WFRFT inverse transform 4 times, and then the results of 4 group processing are obtained. The processing of group $i$ is carried out as follows:

$$
\begin{aligned}
r_{i}^{2}(n)= & F^{-\beta_{i}^{1}}(r(n))=F^{-i+2^{-1}}(r(n)) \\
= & \omega_{0}\left(2^{-1}-i\right) r(n)+\omega_{1}\left(2^{-1}-i\right) R(n) \\
& +\omega_{2}\left(2^{-1}-i\right) r(-n)+\omega_{3}\left(2^{-1}-i\right) R(-n)
\end{aligned}
$$

With the increase of $m$, the priority decreases, and the scan number at level $m$ is $L_{m}=2^{m}$, and $i \in\left[\begin{array}{ll}1 & L_{m-1}\end{array}\right]$. Then the hierarchical rules of (8) are further utilized to get $\beta_{2 i-1}^{m}$ and $\beta_{2 i}^{m}$. Using $\beta_{i}^{2}$, the received signal $r(n)$ is processed by SP4-WFRFT inverse transform $2^{m}$ times, and then the results of the $2^{m}$ group processing are obtained. The processing is conducted as follows:

$$
\begin{aligned}
r_{2 i-1}^{m}(n)= & F^{-\beta_{2 i-1}^{m}}(r(n))=F^{-\beta_{i}^{m-1}+2^{1-m}}(r(n)) \\
= & \omega_{0}\left(-\beta_{i}^{m-1}+2^{1-m}\right) r(n) \\
& +\omega_{1}\left(-\beta_{i}^{m-1}+2^{1-m}\right) R(n) \\
& +\omega_{2}\left(-\beta_{i}^{m-1}+2^{1-m}\right) r(-n) \\
& +\omega_{3}\left(-\beta_{i}^{m-1}+2^{1-m}\right) R(-n) \\
r_{2 i}^{m}(n)= & F^{-\beta_{2 i}^{m}}(r(n))=F^{-\beta_{i}^{m-1}-2^{1-m}}(r(n)) \\
= & \omega_{0}\left(-\beta_{i}^{m-1}-2^{1-m}\right) r(n) \\
& +\omega_{1}\left(-\beta_{i}^{m-1}-2^{1-m}\right) R(n) \\
& +\omega_{2}\left(-\beta_{i}^{m-1}-2^{1-m}\right) r(-n) \\
& +\omega_{3}\left(-\beta_{i}^{m-1}-2^{1-m}\right) R(-n)
\end{aligned}
$$

Furthermore, the following processing can be done when the scanned $\beta_{2 i-1}^{m}$ or $\beta_{2 i}^{m}$ is approximately equal to $\alpha$. Here, let 
$\beta_{2 i}^{m}$ be approximately equal to $\alpha$. Then $r(n)$ is put into (13), and the processing is conducted as follows:

$$
\begin{aligned}
r_{2 i}^{m}(n) & =F^{-\beta_{2 i}^{m}}(r(n))=F^{-\beta_{2 i}^{m}}\left(F^{\alpha}(s(n))+\lambda_{0}(n)\right) \\
& =F^{-\beta_{2 i}^{m}}\left(F^{\alpha}(s(n))\right)+F^{-\beta_{i}^{m}}\left(\lambda_{0}(n)\right) \\
& =F^{\alpha-\beta_{2 i}^{m}}(s(n))+\lambda_{0}^{\prime}(n)
\end{aligned}
$$

Let $\Delta \beta=\alpha-\beta_{2 i}^{m}$; formula (14) can be written as (15) when $\Delta \beta$ is equal to 0 :

$$
r_{2 i}^{m}(n) \approx F^{0}(S(n))+\lambda_{0}^{\prime}(n) \approx S(n)+\lambda_{0}^{\prime}(n)
$$

Because of the norm of the SP-4-WFRFT transform, the statistical characteristic of Gaussian white noise is constant before and after transformation, so it is considered that $\lambda_{0}(n)$ and $\lambda_{0}^{\prime}(n)$ have the same influence. Therefore, in addition to the noise effect, the data signal $s(n)$ can be obtained from signal $r_{2 i}^{m}(n)$, and then $s(n)$ can be demodulated or base-band operation.

The transformation order is scanned from the first to the $M$ level. The scan priority of each level is $m$, the scan sensitivity of each level is expressed by (16), the total scan sensitivity is $2^{1-M}$, and the overall scan number is expressed in (17):

$$
\begin{aligned}
G_{m} & =2^{1-m} \\
L_{s} & =2^{2}+2^{2}+\sum_{m=1}^{M} 2^{m}=2^{3}+\sum_{m=3}^{M} 2^{m}=2^{1+M} .
\end{aligned}
$$

\section{The Research of Performance}

For the convenience of computing, the signal after SP-4WFRFT processing is written as $X$. The statistical mean of its arbitrary order result is $E(X)$. To quantitatively analyze the SP-4-WFRFT processed signal statistical distribution, the zero kurtosis [17] $K$ is defined to compare the degree of convergence of the transformed signal and the Gauss distribution. When $K$ reaches 0 , the signal is more in accord with Gaussian signal characteristics. Then the zero kurtosis $K$ is calculated as

$$
K=\frac{E[X-E(X)]^{4}}{E^{2}[X-E(X)]^{2}}-3 .
$$

Further, by using formula (1), $\left|\omega_{0}\right|^{2}$ is calculated as

$$
\left|\omega_{0}\right|^{2}=\omega_{0} \cdot \omega_{0}^{*}=\cos ^{2}\left(\frac{\Delta \beta \pi}{4}\right) \cos ^{2}\left(\frac{\Delta \beta \pi}{2}\right) .
$$

Then, in the scan process, because the rotational effect of the SP-4-WFRFT will affect the receiver and there is the direct relationship between the effect and weighting coefficient, we define the impact factor $\chi$ as

$$
\begin{aligned}
\chi & =\left|\omega_{0}\right|^{2} \cos ^{2}\left(\frac{-3 \Delta \beta \pi}{4}\right)=\left|\omega_{0} \cos \left(\frac{-3 \Delta \beta \pi}{4}\right)\right|^{2} \\
& =\left|\cos \left(\frac{\Delta \beta \pi}{4}\right) \cos \left(\frac{\Delta \beta \pi}{2}\right) \cos \left(\frac{-3 \Delta \beta \pi}{4}\right)\right|^{2}
\end{aligned}
$$

Here, $\chi$ denotes the response normalized success amplitude in the scan process; $\chi$ is equal to 1 when the scan to $\Delta \beta$ equals 0 , which indicates success.

Under the condition of unknown transformation order, the signal processing by SP-4-WFRFT is equivalent to increasing the noise energy superimposed on the signal, which is equivalent to reducing the signal-to-noise ratio of the receiver. According to the relationship between the equivalent SNR of the receiver under the conditions of the known transformation order and the unknown transformation order, the quantitative formula of the effect of the signalto-noise ratio of the receiver is defined.

The mean power of the signal is defined as $P_{0}$, and the mean power of the noise is defined as $N_{0}$. When the transformation order is known, the SNR of the receiver written as

$$
\mu=10 \cdot \log \left(\frac{P_{0}}{N_{0}}\right) .
$$

For the receiver, when the transformation order is unknown, the equivalent energy is changed to the following:

$$
\begin{aligned}
P^{\prime} & =\chi \cdot P_{0} \\
& =\left|\cos \left(\frac{\Delta \beta \pi}{4}\right) \cos \left(\frac{\Delta \beta \pi}{2}\right) \cos \left(\frac{-3 \Delta \beta \pi}{4}\right)\right|^{2} \cdot P_{0}
\end{aligned}
$$

When the transformation order is unknown, the equivalent SNR of the receiver is expressed as

$$
\begin{aligned}
\mu_{1} & =10 \cdot \log \left[\frac{P^{\prime}}{P_{0}-P^{\prime}+N_{0}}\right] \\
& =10 \cdot \log \left[\frac{\chi}{1-\chi+N_{0} / P}\right] \\
& =10 \cdot \log \left[\frac{\left|\omega_{0} \cos (-3 \Delta \beta \pi / 4)\right|^{2}}{1-\left|\omega_{0} \cos (-3 \Delta \beta \pi / 4)\right|^{2}+10^{-\mu / 10}}\right]
\end{aligned}
$$

This formula shows that the SNR decreases when the transformation order is unknown, which also shows that the bit error rate will be reduced.

\section{Test and Analysis}

5.1. The Performance Analysis. The weighting coefficient is an important parameter in the transformation process, the transformation trend of the weighting coefficient in a main period is shown in Figure 1. It can be seen that the modules of $\omega_{0}, \omega_{1}, \omega_{2}$, and $\omega_{3}$ are periodically transformed in the $0-1$ interval, and $\omega_{l}(\alpha)$ presents a rotation change with the increase or decrease of parameter $\alpha$. Then the alternation between the real and imaginary parts of the weighting coefficient is shown in Figure 2, in which a closed smooth curve can be formed when these four curves are combined. The points on the curve move in a clockwise direction, and the specific order of the change is the following: $\omega_{0}->\omega_{3^{-}}$ $>\omega_{2}->\omega_{1}->\omega_{0}->$. The intersection points of the curve are $(0,0),(0.25,0)$, and $(1,0)$ respectively. 


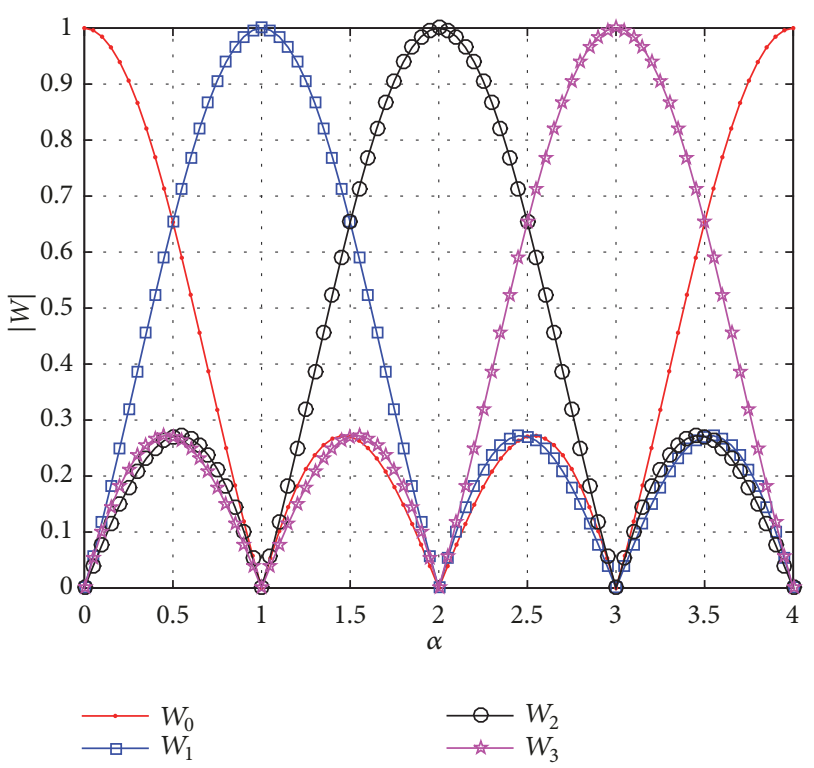

FIGURE 1: Transformation trend of weighting coefficient.

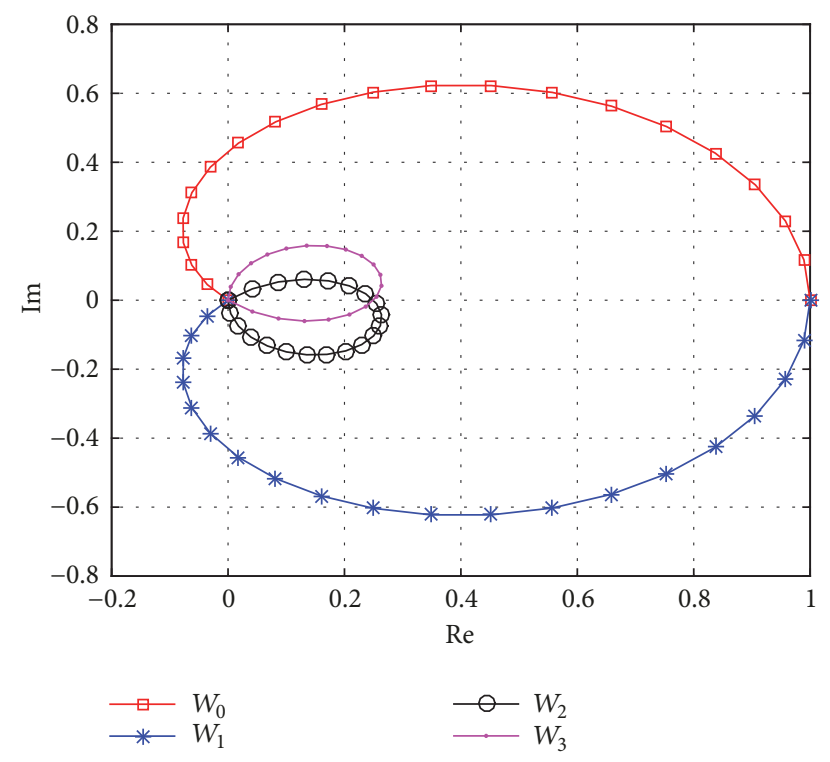

FIGURE 2: Alternation between the real and imaginary parts.

Because the CHS method uses hierarchical technology to scan the transformation order, it was tested in various hierarchical situations. With variation of the hierarchical variable $m$, the changing trend of the scan number of each level $L_{m}$ is shown in Figure 3. The larger the hierarchical variable $m$ is, the greater the scan number $L_{m}$ is, which increases exponentially.

Further, with variation of the hierarchical number $m$, the scan sensitivity $G_{m}$ of each level is shown in Figure 4 . The larger $m$ is, the smaller the scan sensitivity is; that is, the smaller the scan error is, and it decreases exponentially.

Since $\chi$ reflects the normalized amplitude of the scan, the approximate scan is successful when $\chi$ is approximately equal to 1 . Thus, in the scan area, with variation of $\Delta \beta$, the test

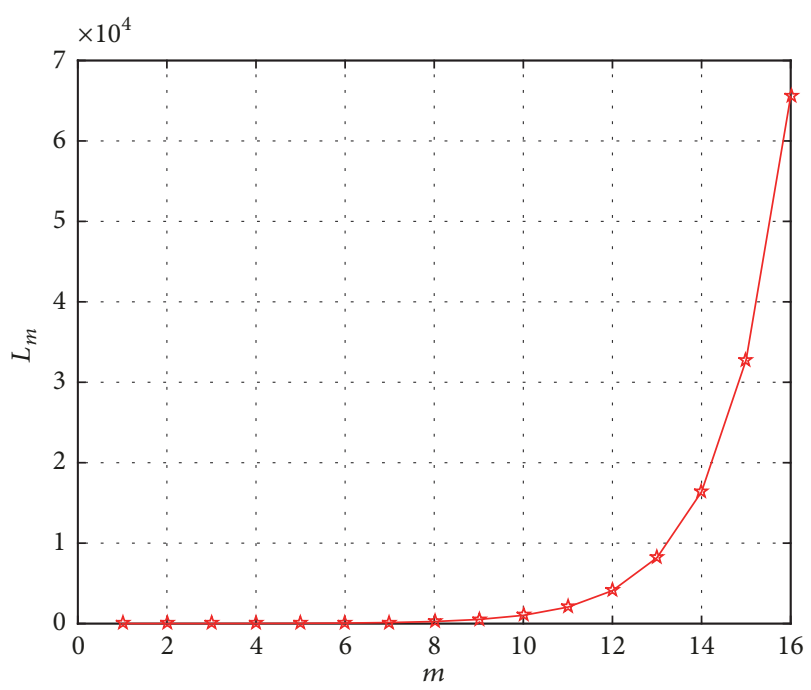

Figure 3: Relationship between $L_{m}$ and $m$.

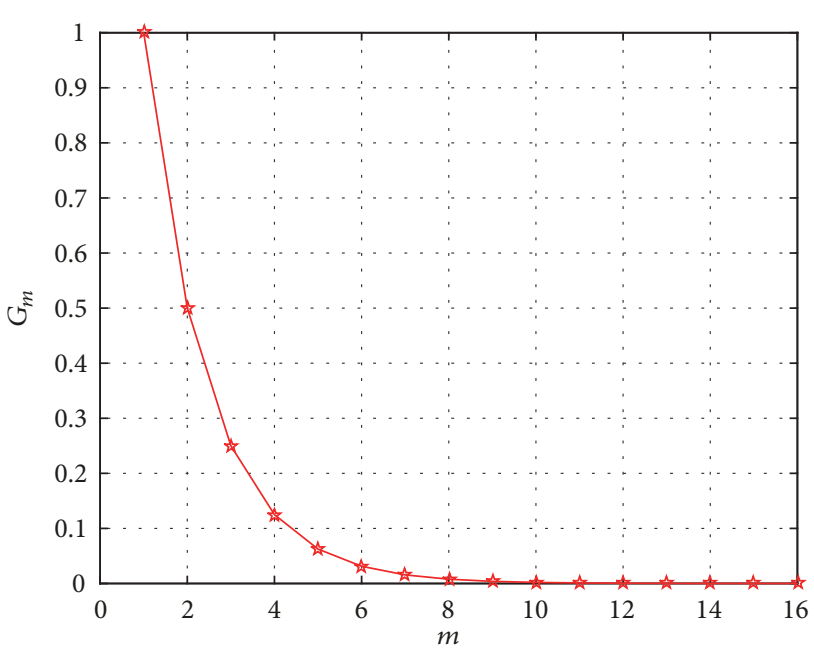

FIGURE 4: Relationship between $G_{m}$ and $m$.

result of influence factor $\chi$ is shown in Figure 5. In the 0-4 main scan period, the requirements of $\chi \approx 1$ can only be met when $\Delta \beta$ is approximately equal to 0 and 4 , which is also consistent with the characteristics of the cycle. The test results of the zeroing kurtosis $K_{p}$ of the communication signals after SP-4-WFRFT transform are shown in Figure 6; the zeroing kurtosis $K_{p}$ is approximately equal to zero. Therefore, the signal is closer to the Gaussian signal, and its transmission concealment has more advantages.

To further analyze the effectiveness of the CHS method, BER (bit error rate) test analysis was carried out. Because the scan value of the transformation order $\beta$ may have error with the real value $\alpha$ of the transmission signal, the BER was assessed with variation of $E_{b} / N_{0}$ under various $\Delta \beta$ conditions. The results are shown in Figure 7 . The BER decreases as $E_{b} / N_{0}$ increases, and, under the same BER condition, the larger $\Delta \beta$ is, the greater the BER is. When $\Delta \beta \leq 0.01$, the BER is close to the ideal condition, which also indicates that further reduction of $\Delta \beta$ will not improve 


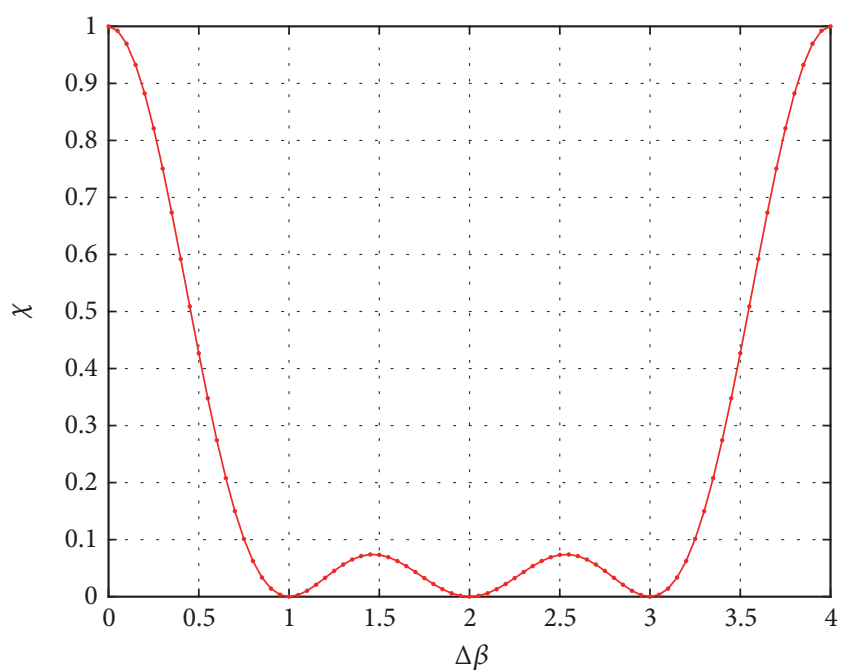

FIGURE 5: Relationship between $\chi$ and $\Delta \beta$.

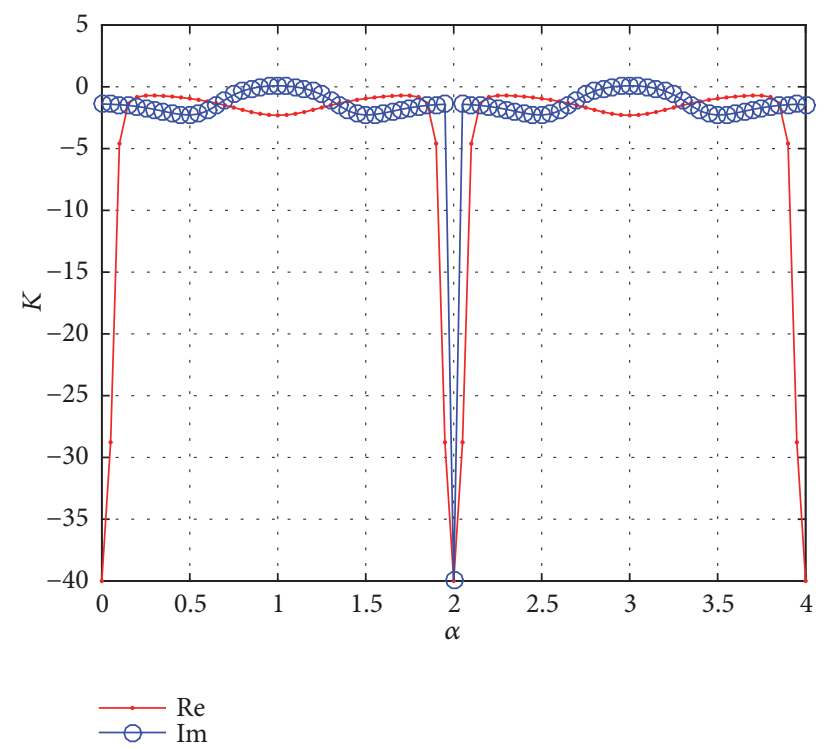

FIgURE 6: Test results of the $K_{p}$.

the BER. For this reason, the maximum scan sensitivity of the transformation order is recommended to be less than 0.01 .

Further, under the minimum $\Delta \beta$ conditions of various $m$ values, with variation of $E_{b} / N_{0}$, the BER calculation results are shown in Figure 8. As seen in the figure, with the increase of $E_{b} / N_{0}$, the BER gradually decreases. Under the same $E_{b} / N_{0}$ condition, the larger the series $m$ is, the smaller the BER is. When $m \geq 8$, the BER is close to ideal, which also indicates that as $m$ further increases, the BER will not improve significantly. To this end, the recommended hierarchical series $m$ value is 8 .

5.2. Comparative Analysis. A comparative analysis of the CHS method and the conventional scanning (CS) method was carried out to verify the superiority of the CHS method. First, the comparison test was conducted with the following

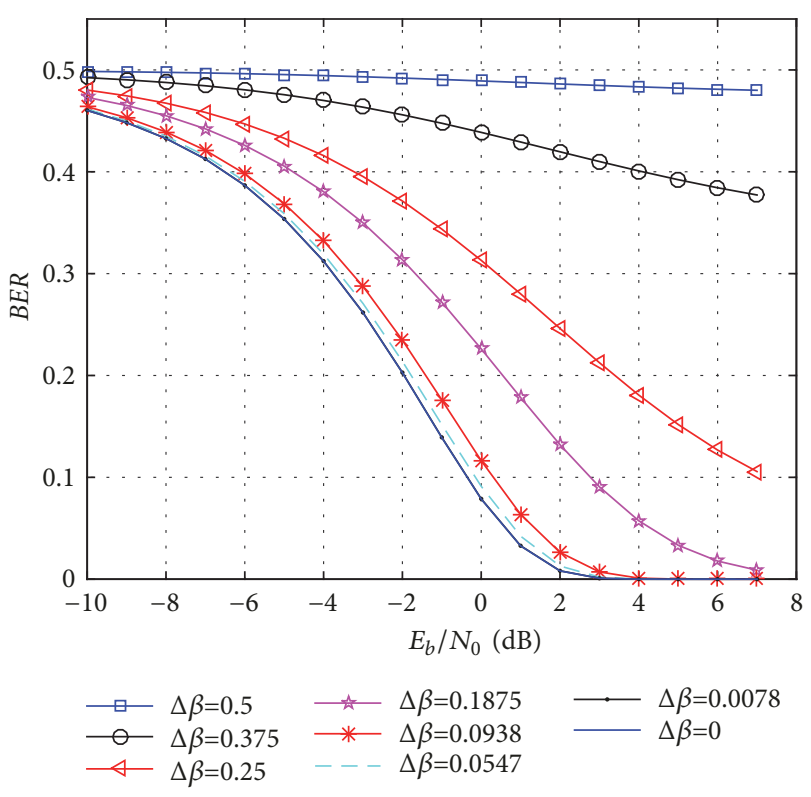

FIGURE 7: BER results with variation of $\Delta \beta$.

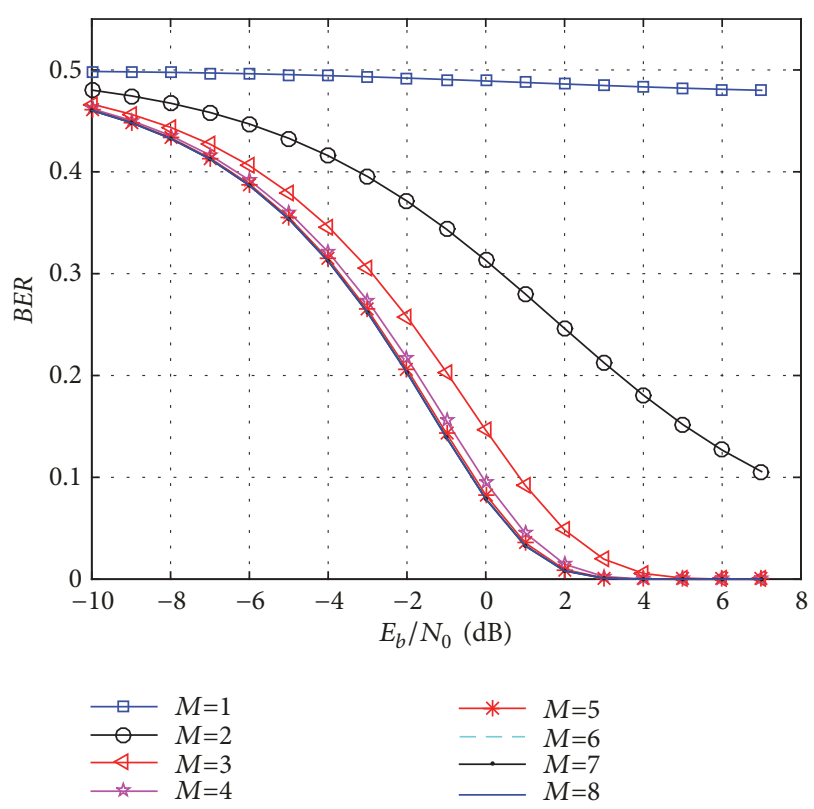

FIGURE 8: BER with variation of $m$.

parameters: the maximum scan number $L_{s}$ of 512 times, the scan sensitivity of the transformation order of 0.0078125 , and the real order $\alpha$ of the transmission signal of 2.2 and 3.35. With increased $L_{s}$, the coverage results of the influence factors are shown in Figures 9 and 10. As seen in the figures, the CS method can only be successful once the scan order is very close to the true value. Because the CHS method performs an overlay scan rather than linear classification, the successful scan area covers more, the probability of success is higher, and the speed of the successful scan is faster. Further statistical analysis shows that the coverage 


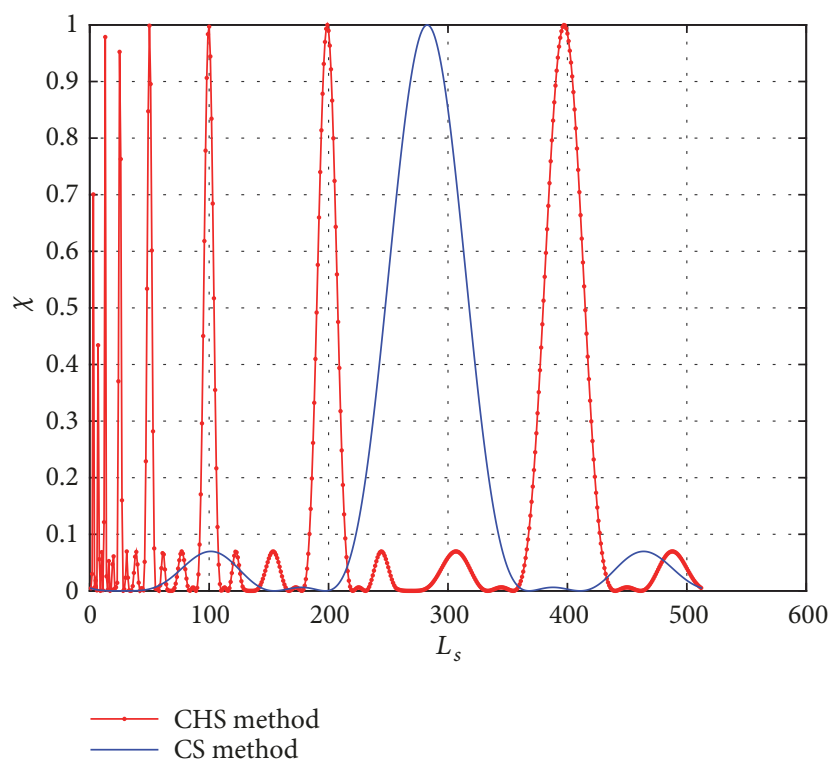

FIGURE 9: Coverage results of the influence factors with $\alpha=2.2$.

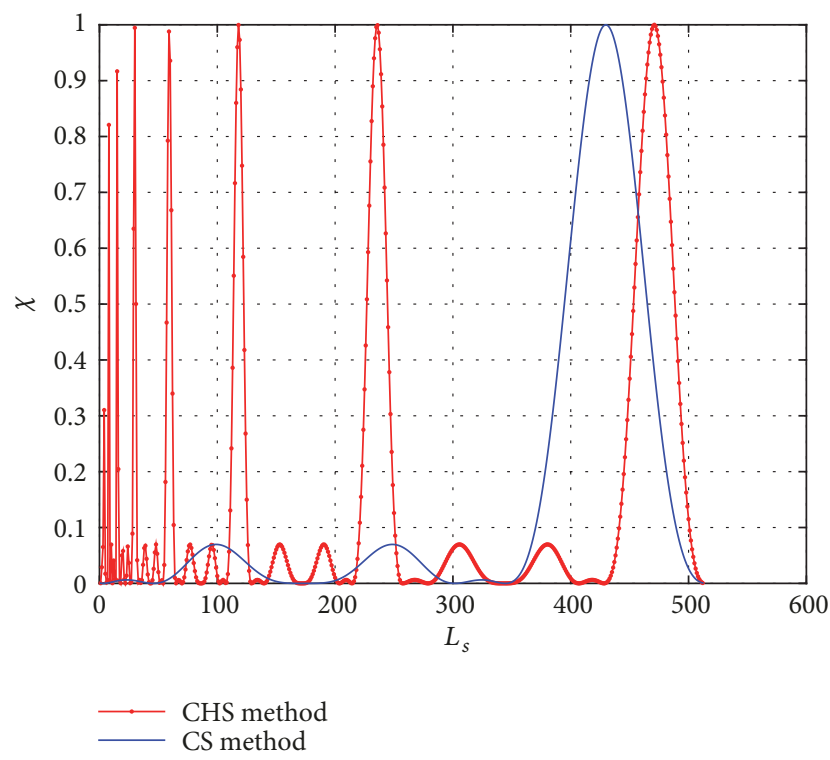

FIGURE 10: Coverage results of the influence factors with $\alpha=3.35$.

comparison results of influencing factors also satisfy this rule under other parameters.

When the overall scan number $L_{s}$ values are 4,16 , and 128 times, respectively, the BER results of the two methods with variation of $E_{b} / N_{0}$ are shown in Figure 11. When the overall scan number $L_{s}$ values are 8, 32, and 256 times, respectively, the BER results of the two methods with variation of $E_{b} / N_{0}$ are shown in Figure 12. They can be seen that the BER of the CS method is relatively large when the scan times are small, while the BER of the CHS method is much smaller. When the scan number is large, the BER of the CS method is improved, but it is still larger than that of the CHS method, which indicates that the BER of the CHS method can meet the requirements better. Further statistical analysis shows that

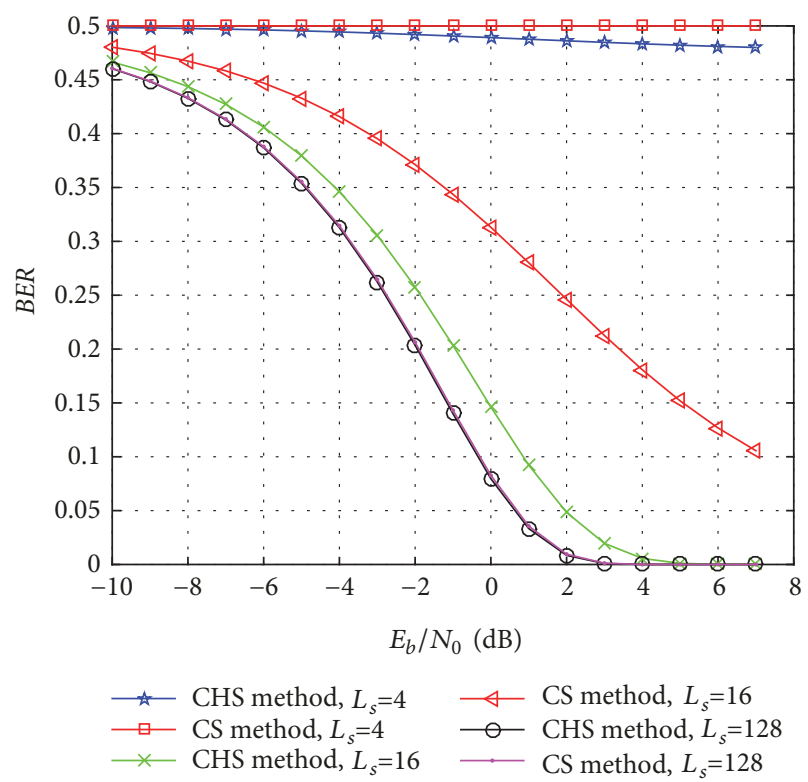

FIgURE 11: The BER results in $L_{s}=4,16$, and 128 .

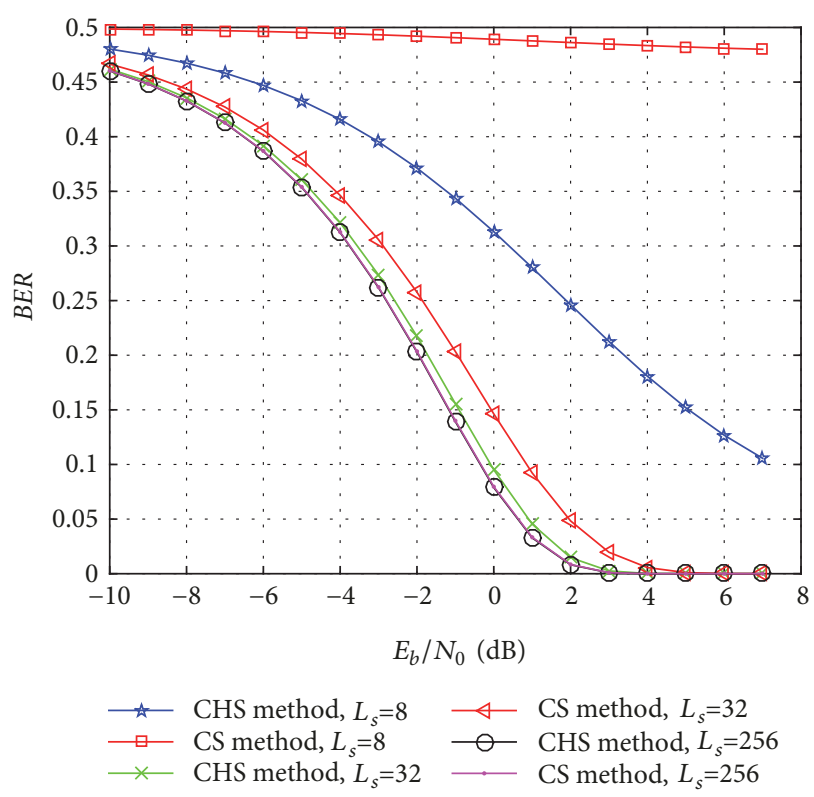

FIgURE 12: The BER results in $L_{s}=8,32$, and 256 .

the BER comparison results also satisfy this rule under other parameters.

With variation of the hierarchical variable $m$, the changing trends of the scan number $L_{s}$ are shown in Figure 13 . The larger the hierarchical variable $m$ is, the greater the scan number $L_{s}$ is, which increases exponentially, and it can be seen that the CHS method has more scanning times than the CS one. Further, with variation of the hierarchical number $m$, the scan sensitivity $G_{m}$ is shown in Figure 14 . The larger $m$ is, the smaller the scan sensitivity is, that is, the smaller the scan error is, and it decreases exponentially, and the sensitivity of the CHS method is higher than that of the CS method. The 


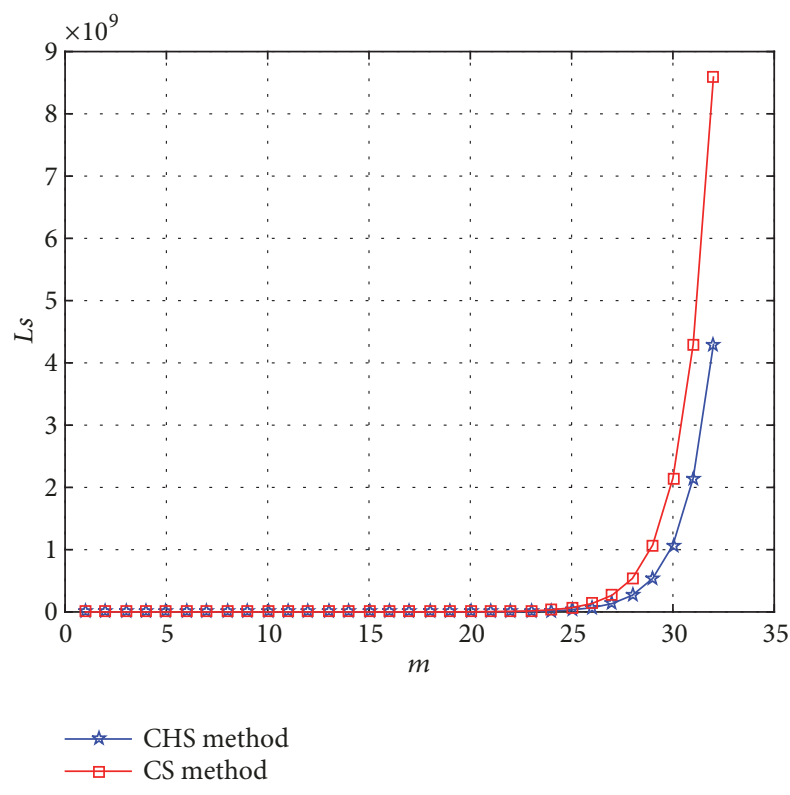

FIGURE 13: The comparison result of the scan number.

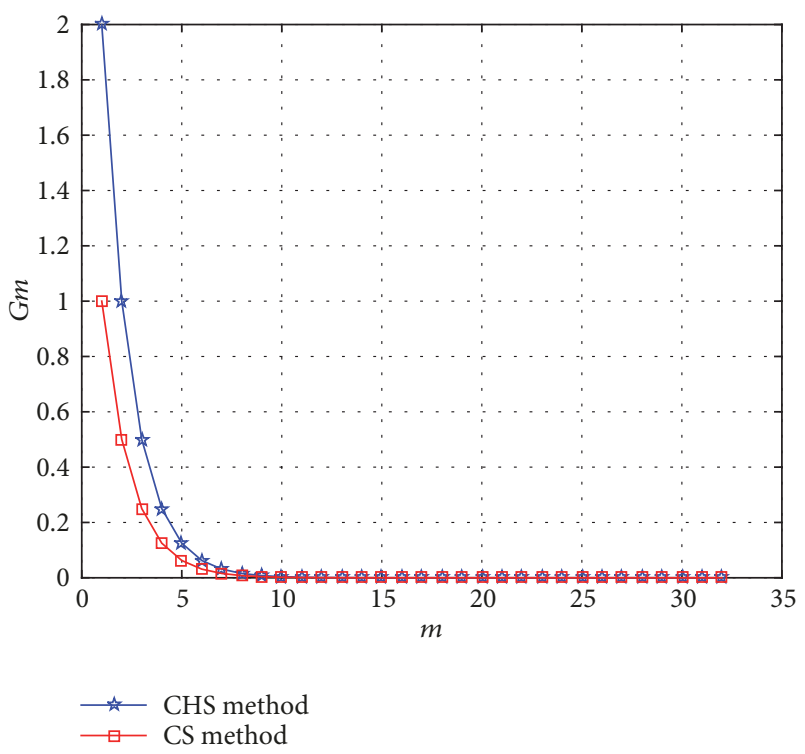

FIGURE 14: The comparison result of the scan sensitivity.

results show that the scanning precision of the CHS method is higher.

\section{Conclusion}

Aiming at the characteristics of a digital communication system based on SP-4-WFRFT, a cross-hierarchical scanning (CHS) method was proposed. The test results show that the larger the hierarchical variable $m$ is, the greater the scan number $L_{m}$ is, the smaller the scan sensitivity is, and the smaller the scan error is, which is in accordance with the theory. Then the larger $\Delta \beta$ is, the greater the BER, and the $\mathrm{BER}$ is close to the ideal condition when $\Delta \beta \leq 0.01$. The larger the series $m$ is, the smaller the BER is, and the BER is close to ideal when $m \geq 8$. Thus, the maximum scan sensitivity of the transformation order is recommended to be less than 0.01, and the recommended hierarchical series $m$ value is 8 . Further, a comparative analysis of the CHS method and CS method was carried out. The result shows that CHS method has a larger successful scan area and higher success probability. Moreover, the BER of the CHS method is smaller than that of the CS method, which indicates that the BER of the CHS method can meet the requirements better. Further statistical analysis shows that the comparison results also satisfy this rule under other parameters. Furthermore, the sensitivity of the CHS method is higher than that of the CS method. The results show that the scanning precision of the CHS method is higher.

\section{Data Availability}

The data is generated by using the formula principle and simulated in MATLAB software. The type of data is floatingpoint. The data used to support the findings of this study are currently under protection. Requests for data, [6/12 months] after publication of this article, will be considered by the corresponding author.

\section{Disclosure}

The author declares that all the data in this paper are real and available.

\section{Conflicts of Interest}

The author declares that they have no conflicts of interest.

\section{Acknowledgments}

This work was financially supported by the National Natural Science Foundation of China (Grant no. 61501309), the China Postdoctoral Science Foundation (Grants nos. 2017T100185 and 2015M580231), and the Liaoning Natural Science Foundation of China (Grant no. 2017011002-301).

\section{References}

[1] N. Wiener, "Hermitian Polynomials and Fourier Analysis," Journal of Mathematics and Physics, vol. 8, no. 1-4, pp. 70-73, 1929.

[2] V. Namias, "The fractional order Fourier transform and its application to quantum mechanics," Journal of Applied Mathematics, vol. 25, no. 3, pp. 241-265, 1980.

[3] A. C. McBride and F. H. Kerr, "On Namias's fractional Fourier transforms," IMA Journal of Applied Mathematics, vol. 39, no. 2, pp. 159-175, 1987.

[4] A. W. Lohmann, "Image rotation, wigner rotation, and the fractional fourier transform," Journal of the Optical Society of America A: Optics and Image Science, and Vision, vol. 10, no. 10, pp. 2181-2186, 1993.

[5] C.-C. Shih, "Fractionalization of Fourier transform," Optics Communications, vol. 118, no. 5-6, pp. 495-498, 1995. 
[6] M. A. Kutay, H. Özaktaş, H. M. Ozaktas et al., "Optimal Filtering in Fractional Fourier Domains," in Proceedings of the IEEE International Conference on Acoustics, Speech \& Signal Processing, pp. 937-940, IEEE, 1995.

[7] S. Liu, J. Zhang, and Y. Zhang, "Properties of the fractionalization of a Fourier transform," Optics Communications, vol. 133, no. 1-6, pp. 50-54, 1997.

[8] G. Cariolaro, T. Erseghe, P. Kraniauskas, and N. Laurenti, "A unified framework for the fractional Fourier transform," IEEE Transactions on Signal Processing, vol. 46, no. 12, pp. 3206-3219, 1998.

[9] J. Lang, R. Tao, Q. Ran, and Y. Wang, "The multiple-parameter fractional Fourier transform," Science China Information Sciences, vol. 51, no. 8, pp. 1010-1024, 2008.

[10] P. Oonincx and B. Muth, "A fractional fourier based correlator for detecting joint time and frequency offsets," in Proceedings of the 2007 15th International Conference on Digital Signal Processing, DSP 2007, pp. 623-626, July 2007.

[11] H. Mingjun, W. Yongmin, and N. Jiahong, "High- Dynamic Target Detection Method Based on FRFT," Journal of Detection \& Control, vol. 38, no. 1, pp. 37-41, 2016.

[12] Z. Zhang, X. Da, and H. Liu, "A study of the covered characteristics of MAP- WFRFT satellite signals," Journal of Chongqing University of Posts and Telecommunications (Natural Science Edition), vol. 29, no. 4, pp. 460-467, 2017.

[13] X.-J. Sha, X.-J. Fang, and L. Mei, "Novel Anti-recognition Method Based on Weighted Fractional Fourier Transform," Radio Communications Technology, vol. 42, no. 3, pp. 1-4, 2016.

[14] M.-F. Lu, F. Zhang, G.-Q. Ni, and R. Tao, "Analysis and improvement of actual sampling system in fractional Fourier transform domain," Journal of Electronics and Information Technology, vol. 35, no. 9, pp. 2094-2099, 2013.

[15] R. Wang and Y. Ma, "DOA estimation of wideband linear frequency modulated pulse signals based on fractional Fourier transform," Acta Armamentarii, vol. 35, no. 3, pp. 421-427, 2014.

[16] M. Lin, Weighted-type Fractional Fourier Transform and its Applications in Communication Systems, vol. 10, Harbin Institute of Technology, 2010.

[17] O. Wenxiang, Research on Performance of Low-probability-ofdetection Signal based on Fractional Fourier domain, vol. 6, Harbin Institute of Technology, 2016. 


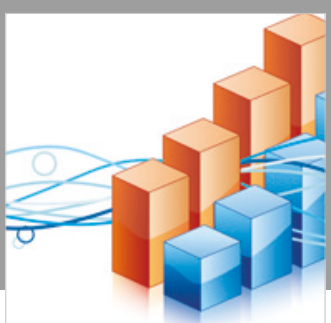

Advances in

Operations Research

\section{-n-m}
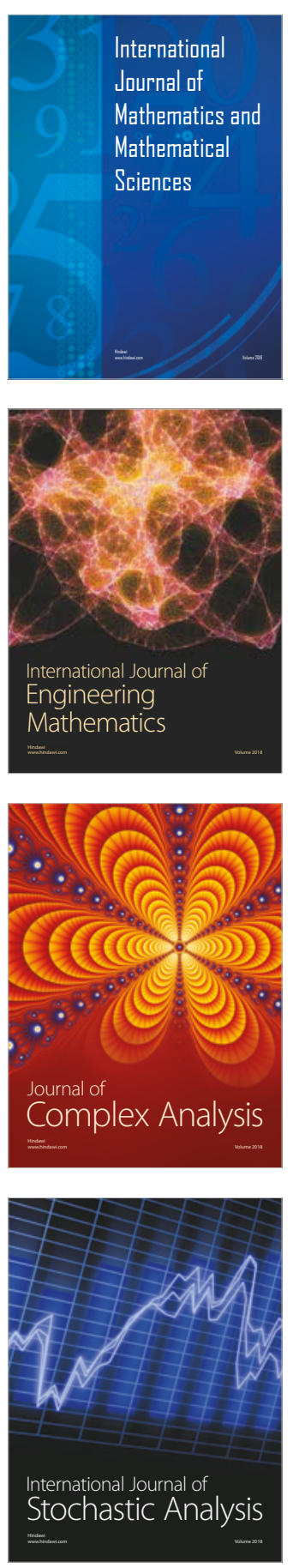
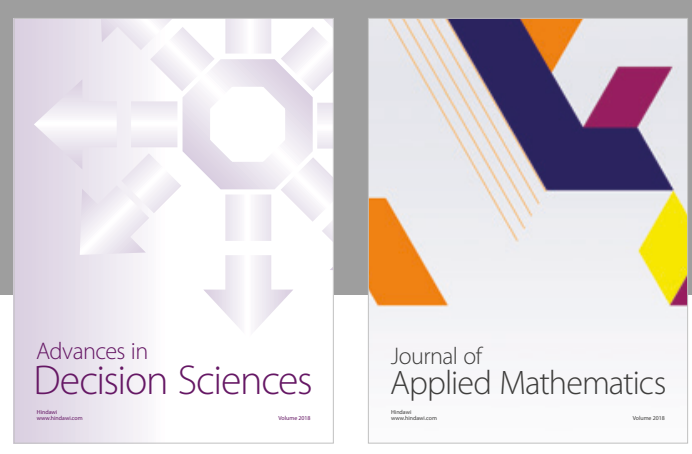

Journal of

Applied Mathematics
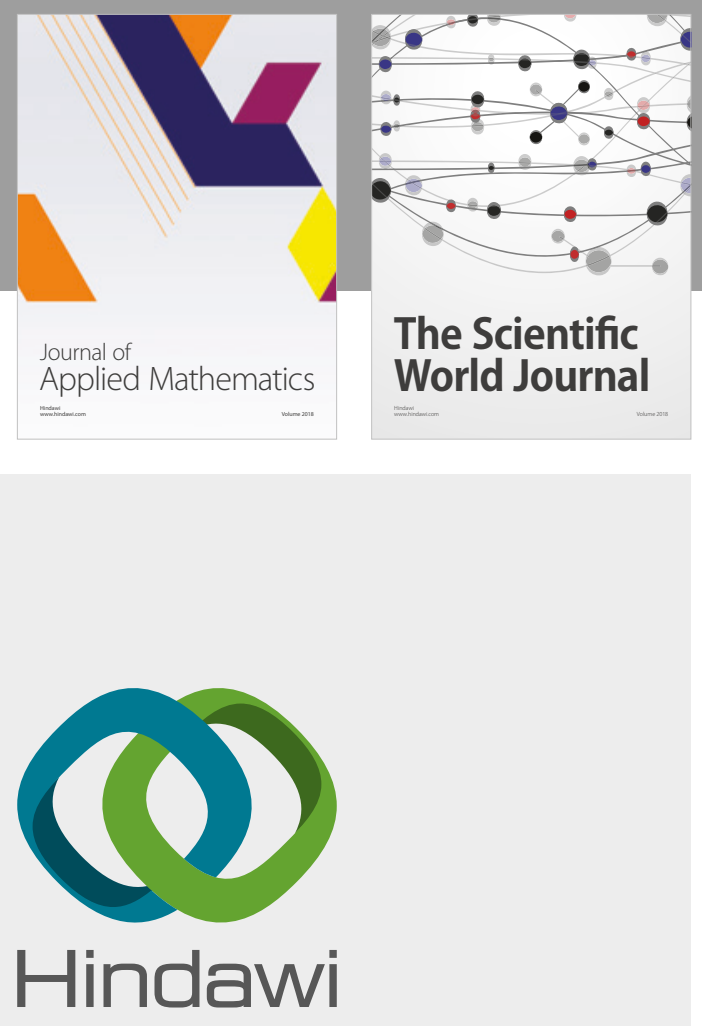

Submit your manuscripts at

www.hindawi.com

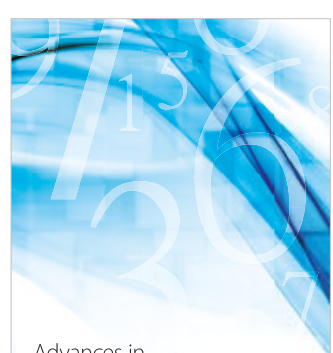

Advances in
Numerical Analysis
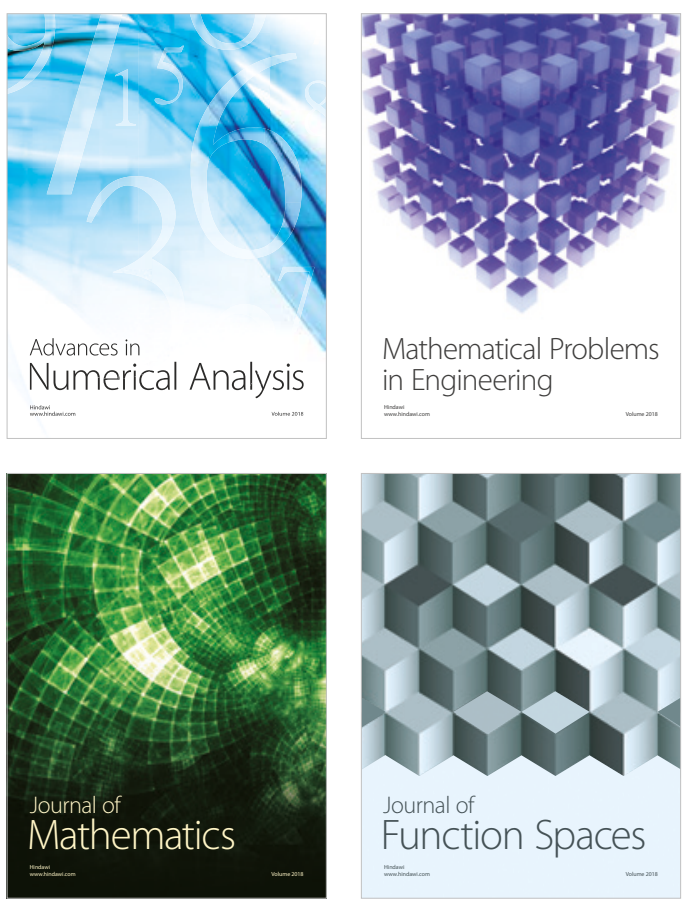

Mathematical Problems in Engineering

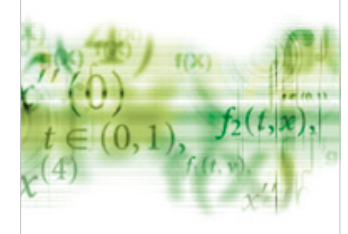

International Journal of

Differential Equations

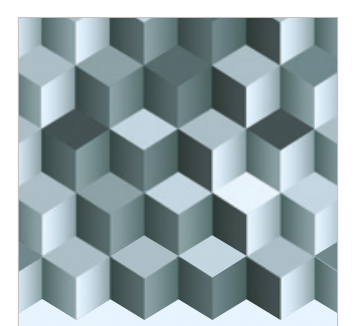

Journal of

Function Spaces

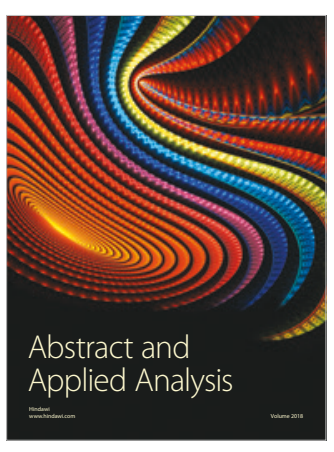

The Scientific

World Journal

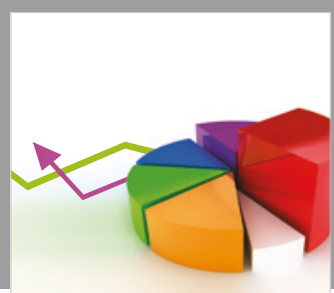

Journal of

Probability and Statistics
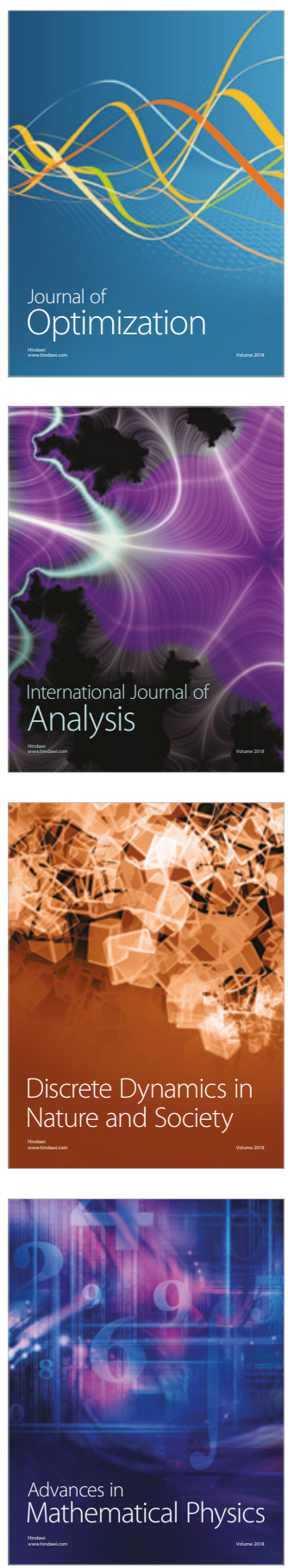